bad ones; good equipment and premises cost no more, and in the long run much less, than bad ones."

Within clinical laboratories the laboratory manager is responsible for ensuring that safety policies are written down and practised by all who work there. There is an increasing trend, however, to have equipment available in other clinical areas to provide rapid results or for emergency testing - for example, reflectometers in outpatient clinics and blood gas analysers in intensive care units. These areas should be regarded as extensions of the clinical laboratory, and it must be made perfectly clear who is responsible for the apparatus and for training and supervising the staff using it. ${ }^{9}$ The further extension of side room testing to community based clinics and general practitioners' surgeries implies that the dangers of infection from biological specimens need to be widely known. It also needs to be made clear who is responsible-and thus answerable for accidents-in these settings.

One aspect of the code that does require emphasis and wide dissemination is the onus it places on the person who takes specimens and sends them to the laboratory to make sure they are safe to handle and that any known risk is identified. This does not mean that all risks will be identified as new bloodborne infections continue to be discovered.
Despite the caveats about overrestrictive interpretation safety at work is of paramount importance in the health service and the updated code is a welcome contribution. The code takes the form of three documents-on clinical laboratories, on the mortuary and postmortem room, and model rules for staff and visitors. Health authorities are responsible for seeing that laboratories and mortuaries conform to the new code, but the model rules should be essential reading for all health service staff.

Consultant Microbiologist,

University College Hospital,

London WC1E 6AU

1 DHSS. Code of practice for the prevention of infection in clinical laboratories and post mortem rooms. London: HMSO, 1978.

2 Health and Safety Commission's Health Services Advisory Committee. Safe working and the prevention of infection in clinical laboratories. London: HMSO, 1991.

3 Health and Safety Commission's Health Services Advisory Committee. Safe working and the prevention of infection in the mortuary and post-mortem room. London: HMSO, 1991.

4 Health and Safety Commission's Health Services Advisory Committee. Safe working and the prevention of infection in clinical laboratories-model rules for staff and visitors. London: HMSO, 1991.

5 White C. Safety guidelines for laboratories. BMf 1991;303:808

6 Howie JH, Collins $\mathrm{CH}$. The Howie Code for preventing infections in clinical laboratories: comments on some general criticisms and specific complaints. BMJ 1980;200:1071-4.

Collins CH, Howie JH. The Howie Code: what are the real costs? 7 Clin Pathol 1984:37:83-6.

7 Collins $\mathrm{CH}$, Howie JH. The Howie Code: what are the real costs? $\mathrm{f} \mathrm{Clin}$

8 Collins CH. Laboratory-acquired infections. London: Butterworth, 1983. diagnostic laboratory. Cardiff: Welsh Scientific Advisory Committee, 1984. (WSAC/D/1/84.)

\title{
Using tympanometry to detect glue ear in general practice
}

\section{Overreliance will lead to overtreatment}

The most common use of tympanometry is to show the negative middle ear pressure associated with otitis media with effusion. It is non-invasive, rapid to perform, and well accepted even by young children and infants. With the introduction of portable and, more recently, hand held equipment tympanometry has become widely available and cheap to perform. Should general practitioners be using tympanometry to detect otitis media with effusion?

Tympanometry measures the proportion of an acoustic signal transferred from the external to the internal ear and compares the absorbed and reflected components to indicate middle ear pressure. Comparison with findings at myringotomy shows that the technique has a high sensitivity (about $90 \%$ ) and specificity (about $75 \%)^{1}$ : most children with otitis media with effusion show a flat, low compliance trace (type B) and very few the normal symmetrical peaked curve (type A). Nevertheless, these results may be no better than those obtainable by a well trained clinician using pneumatic otoscopy to examine the ear. Some training in using the tympanometer is necessary because an inadequate seal in the meatus may produce a flat trace which is then wrongly interpreted as showing middle ear fluid.

At present tympanometry is used both for screening and for diagnosis by audiological technicians. Since tympanometry cannot distinguish marginal from mild to moderate hearing $\operatorname{loss}^{2}$ it is usually combined with an assessment of hearing loss. Our district's pattern of referral for otitis media with effusion may be universal: most cases are first suspected by parents, and the children are referred to hospital after a period of observation with otoscopy, tympanometry, and hearing assessment by senior clinical medical officers in audiology. de Melker suggests that general practitioners using a hand held microtympanometer can also reliably diagnose otitis media with effusion ( $\mathrm{p} 96)$.
The incidence and clinical course of otitis media in childhood are reasons for caution about wider, uncontrolled use of tympanometry. The incidence of glue ear peaks during the second half of the first year of life and declines gradually thereafter. ${ }^{4}$ Twenty to thirty per cent of children under 6 develop the condition, but prevalence rates vary according to the diagnostic techniques used. Ninety per cent of effusions after otitis media resolve within three months. In a study of bilateral disease 322 children referred to hospital were assessed to produce a cohort of 150 in whom the disease had remained constant in each ear for three months before surgery. ${ }^{5}$ Even in the unoperated ears almost $30 \%$ of the effusions had resolved within a year after surgery to the contralateral ear. Thus even in severe cases there is a high spontaneous resolution rate that is mainly age related.

Nevertheless, in 1985 in England and Wales about 60000 children were treated for otitis media with myringotomy or grommets. A further 16000 underwent adenoidectomies, some of which may have been for ear disease. A recent survey showed that $64 \%$ of otolaryngologists waited for a period to confirm the persistence of middle ear fluid before listing the child for surgery $-71 \%$ of them for two months or more. ${ }^{6}$ Eighty five per cent of surgeons used tympanometry for diagnosing otitis media with effusion, as well as otoscopy and audiometry. A recent study from Oxford, however, showed that $34 \%$ of children undergoing surgery for otitis media with effusion were found not to have middle ear fluid.

The danger of using tympanometry more widely in general practice is that its high sensitivity for detecting otitis media with effusion together with the high rate of spontaneous resolution may lead to over-referral of mild cases. Failure to wait and retest at each stage may lead to overtreatment. ${ }^{8}$ Tympanometry can undoubtedly help general practitioners improve their diagnostic ability in middle ear disease, but it 
should not supplant careful examination of the tympanic membrane with a pneumatic otoscope-which may also detect other important conditions missed by tympanometry, such as erosion of the outer attic wall, atelectasis, and cholesteatoma.

Consultant Ear, Nose, and Throat Surgeon,

A RICHARD MAW

Bristol Royal Infirmary,

Bristol BS2 8HW

1 Brooks DN. Acoustic impedance studies on otitis media with effusion. Int $f$ Pediatr Otorhinolaryngol 1982:4:89-94
2 Fiellau-Nikolajsen M. Tympanometry and secretory otitis media. Acta Otolaryngol (Stockh) 1983;394(suppl):1-72.

3 de Melker RA. Diagnostic value of microtympanometry in primary care. BMf 1991;303:96-8.

4 Pukander J. Acute otitis media among rural children in Finland. Int $\mathcal{F}$ Pediatr Otorhinolaryngol 1982;4:325-32.

Maw AR, Herod F. Otoscopic impedance and audiometric findings in glue ear treated by adenoidectomy and tonsillectomy. A prospective randomised study. Lancet 1986;ii:1399-402. Smith IM, Maw AR. Secretory otitis media: a review of management by consultant otolaryngologists. Clin Otolaryngol 1991;16:266-70.

Black N, Crowther J, Freeland A. The effectiveness of adenoidectomy in the treatment of glue ear: randomized controlled trial. Clin Otolaryngol 1986;11:149-55

8 Lous J. Screening for secretory otitis media: evaluation of some impedance screening programs for long-lasting secretory otitis media in 7 year old children. Int $\mathcal{f}$ Pediatr Otorhinolaryngol long-lasting secret $1987 ; 13: 85-97$.

9 Reves R, Budgett R, Miller D, Wadsworth J, Haines A. Study of middle ear disease using tympanometry in general practice. BMJ 1985;290:1953.

\section{Bone banks}

\section{Could be held by regional blood transfusion centres}

Bone grafts are used by a variety of surgeons-orthopaedic; faciomaxillary; dental; ear, nose, and throat; and neurosurgeons - for a variety of purposes: to fill bone cysts; for cervical fusion, facial and limb reconstruction after resection of tumours, and hip revision; and to aid the healing of ununited fractures. ${ }^{12}$ Bone grafts may be either autologous or allogeneic. Allogeneic grafts have many advantages, not least the fact that they do not necessitate additional surgery to obtain the bone, but their efficient use depends on good facilities for keeping the bone until it is needed. Bone allografts not only avoid all the potential operative complications and costs of autografts; they also offer the advantage of an unlimited amount of bone of varying shapes for transplantation. The grafting of donor's bone is not compromised by crossing the HLA, ABO, or Rh (D) barriers, and immunosuppressive treatment is not needed, although recipients may develop HLA antibodies and $\mathrm{Rh}$ negative recipients may produce anti-D.

The most commonly used bone allografts from live donors are femoral heads excised during hip replacement operations. These bones are quarantined until the donors have been confirmed to be free of transmissible diseases (AIDS, hepatitis $\mathrm{B}$, hepatitis $\mathrm{C}$, and syphilis) by serological testing six months after donation. Bones from dead donors may be banked, but the freedom from infection cannot be confirmed. Nevertheless, modern diagnostic techniques such as the polymerase chain reaction may overcome this drawback.

Selecting the right bone donor is as important as selecting the right blood donor. Even the exclusion criteria are almost identical. These include a history of metabolic bone disorder or disease of malignant, autoimmune, or unknown cause; active systemic infection or transmissible infectious disease; belonging to a high risk group for HIV infection; and prolonged treatment with steroids, exposure to toxins, or irradiation to the relevant bones.

Bone banks have been established through different routes by various local initiatives. Some are part of independent institutions that handle a variety of tissues, while others belong to hospital orthopaedic departments. To maintain standards of procurement, processing, storage, administration, and documentation, however, reliable organisations with experienced staff are required. Inevitably, the operational overhead is beyond the capability of most hospitals, and the investment and expense can be justified only for a bank that serves many hospitals.

Regional blood transfusion centres are ideally suited to house bone banks where these do not already exist. The centres have over 50 years' experience in recruiting and selecting donors, laboratory testing, storage know how, research programmes, and quality assurance systems, as well as a network of communications and transportation to all hospitals in their regions. They are staffed around the clock and have links with local and national transplant coordinators through their tissue typing departments. All these ingredients are essential for the success of any bone bank.

The maintenance of effective cooperation with the main suppliers and users of bone grafts - orthopaedic surgeons and skilful public relations efforts, directed towards potential donors and their families and the general public, are also necessary. Moreover, regional bone banks within the Blood Transfusion Service could, in time, expand and act as a nucleus for truly comprehensive tissue banks (holding cornea, skin, and pancreatic islets), thus combining cost effectiveness with a better and more comprehensive service to patients and the community.

Consultant Haematologist in Transfusion Medicine,

West Midlands Regional Blood Transfusion Service, Birmingham B15 2SG

1 Jackson BJ. Bone banking: an overview. Laboratory Medicine 1987;18:830-3.

2 Kahn RA. Establishing a tissue bank in a blood collection facility: an introduction to bone banking. In: Glassman $A B$, Umlas J, eds. Cryopreservation of tissue and solid organs for transplantation. Virginia: American Association of Blood Banks, 1983:13-28. 